\title{
Improved Worst-Case Deterministic Parallel Dynamic Minimum Spanning Forest
}

\author{
Tsvi Kopelowitz \\ Bar-Ilan University \\ Ramat-Gan, Israel \\ kopelot@gmail.com
}

\author{
Ely Porat \\ Bar-Ilan University \\ Ramat-Gan, Israel \\ porately@cs.biu.ac.il
}

\author{
Yair Rosenmutter \\ Bar-Ilan University \\ Ramat-Gan, Israel \\ yapoke@gmail.com
}

\begin{abstract}
This paper gives a new deterministic algorithm for the dynamic Minimum Spanning Forest (MSF) problem in the EREW PRAM model, where the goal is to maintain a MSF of a weighted graph with $n$ vertices and $m$ edges while supporting edge insertions and deletions. We show that one can solve the dynamic MSF problem using $O(\sqrt{n})$ processors and $O(\log n)$ worst-case update time, for a total of $O(\sqrt{n} \log n)$ work. This improves on the work of Ferragina [IPPS 1995] which costs $O(\log n)$ worst-case update time and $O\left(n^{2 / 3} \log \frac{m}{n}\right)$ work.
\end{abstract}

\section{ACM Reference Format:}

Tsvi Kopelowitz, Ely Porat, and Yair Rosenmutter. 2018. Improved WorstCase Deterministic Parallel Dynamic Minimum Spanning Forest. In SPAA '18: 30th ACM Symposium on Parallelism in Algorithms and Architectures, fuly 16-18, 2018, Vienna, Austria. ACM, New York, NY, USA, 9 pages. https: //doi.org/10.1145/3210377.3210403

\section{INTRODUCTION}

In the dynamic minimum spanning forest (MSF) problem, the goal is maintain a MSF $F$ of an undirected dynamic graph $G=(V, E)$ with weight function $w: E \rightarrow \mathbb{R}$, while supporting edge insertions and deletions. The dynamic MSF problem is one of the most fundamental dynamic graph problems, and has been used as a subroutine for solving many other graph problems ([1],[3],[21],[22]). The first sequential algorithm solving the dynamic MSF problem has a worstcase update time of $O(\sqrt{m})$, where $m$ is the number of edges, and was introduced by Frederickson [6]. Using the sparsification technique of Eppstein et al. [3, 4] on Fredrickson's algorithm reduces the worst-case update time to $O(\sqrt{n})$, where $n$ is the number of vertices. Both of these results are deterministicWhile there have been several improvements on the time cost when using randomization or allowing amortization, the $O(\sqrt{n})$ time bound is the best known for deterministic worst-case dynamic MSF.

Dynamic MSF in the PRAM model. While the dynamic MSF problem in sequential models has received a lot of attention from researchers, there has been no progress since the 90s in the PRAM Model. Das and Ferragina [2] presented a dynamic MSF algorithm

Permission to make digital or hard copies of all or part of this work for personal or classroom use is granted without fee provided that copies are not made or distributed for profit or commercial advantage and that copies bear this notice and the full citation on the first page. Copyrights for components of this work owned by others than ACM must be honored. Abstracting with credit is permitted. To copy otherwise, or republish, to post on servers or to redistribute to lists, requires prior specific permission and/or a fee. Request permissions from permissions@acm.org.

SPAA '18, fuly 16-18, 2018, Vienna, Austria

(c) 2018 Association for Computing Machinery.

ACM ISBN 978-1-4503-5799-9/18/07 . \$15.00

https://doi.org/10.1145/3210377.3210403 in the EREW PRAM model, which is based on Frederickson's [6] sequential algorithm, that uses $O\left(\frac{m^{2 / 3}}{\log n}\right)$ processors, $O(\log n)$ worstcase time, and $O\left(\mathrm{~m}^{2 / 3}\right)$ work. Ferragina [5] showed how to parallelize the sparsification technique of Eppstein et al. ([3], [4]), thereby obtaining a fully dynamic MSF algorithm in the EREW PRAM model, that uses $O\left(\frac{n^{2 / 3} \log \frac{m}{n}}{\log n}\right)$ processors, $O(\log n)$ worstcase time, and $O\left(n^{2 / 3} \log \frac{m}{n}\right)$ work. Liang and McKay [16] proposed a different parallel algorithm for dynamic MSF that uses $O\left(n^{2 / 3}\right)$ processors and has $O\left(\log n \cdot \log \frac{m}{n}\right)$ parallel worst-case time.

Our results. In this paper we give the first improvement on dynamic MSF in the EREW PRAM model in over 20 years (in terms of deterministic worst-case update times). The main result is summarized by the following theorem.

THEOREM 1.1. There exists a deterministic algorithm for the $d y$ namic MSF problem in the EREW PRAM model that uses $O(\sqrt{n})$ processors and has a parallel worst-case update time of $O(\log n)$. The resulted work of the algorithm is $O(\sqrt{n} \log n)$.

Dynamic MSF and Dynamic Connectivity. In the dynamic connectivity problem the goal is to maintain a dynamic graph $G$ with edge insertions and deletions, while supporting connectivity queries: "given two vertices in $G$, are the vertices in the same connected component?". The dynamic connectivity problem is a weaker version of the dynamic MSF problem, since one way of solving dynamic connectivity is to maintain a spanning forest of $G$ and using dynamic connectivity data structures for forests such as [20]. Thus, Frederickson's algorithm together with the sparsification techinque yield a $O(\sqrt{n})$ worst-case deterministic update time for dynamic connectivity. Recently, Kejlberg-Rasmussen et al. [14] reduced the runtime slightly to $O\left(\sqrt{\frac{n(\log \log n)^{2}}{\log n}}\right)$. The proof of Theorem 1.1 is based on the approach used by Kejlberg-Rasmussen et al. [14] for solving dynamic connectivity.

\subsection{Algorithmic Overview}

Throughout the paper we apply the standard assumption that the graph is sparse, i.e., the graph has $m=O(n)$ edges. In the sequential case this assumption is permissable due to the sparsification technique of [4]. We later show how to extend the sparsification technique for dynamic MSF to the EREW PRAM model. We also assume throughout the paper that the maximum degree in $G$ is 3 by applying the techniques of Frederickson [6]. This last assumption costs an $O(1)$ worst-case time additive overhead per operation.

We are now ready to provide an overview of our techniques. We emphasize that our overview sacrifices accuracy for the sake of 
intuition. An accurate description of the techniques that we use is given in the rest of the paper. We believe it is best to first discuss a sequential version of our dynamic MSF algorithm, which is an $O(\sqrt{\log n})$ factor slower than the algorithm of Frederickson [6] after applying the sparsification technique of [4]. Nevertheless, the proof of the following theorem is helpful for understanding our proof of Theorem 1.1.

Theorem 1.2. There exists a sequential deterministic algorithm for the dynamic MSF problem, which has a worst-case update time of $O(\sqrt{n \log n})$.

Euler tours and lists. The proof of Theorem 1.2 is based on the dynamic connectivity algorithm presented in [14]. The basic technique is to maintain Euler tours of the trees in $F$, which is the MSF of $G$. An Eulerian circuit in a directed graph is a tour on the edges of the graph, starting and ending on the same vertex, in which each edge is visited exactly once. There is an Eulerian circuit if and only if for every vertex $u$ in the graph the out-degree of $u$ is the same as the in-degree of $u$. For a tree $T$ in a spanning forest of an undirected graph $G$, an Euler tour of $T$ is a list of the edges in $T$. The Euler tour of $T$ is created by treating each undirected edge as two directed edges in different directions, so that for every vertex $u$ in $T$ the out-degree of $u$ is the same as the in-degree of $u$. The authors of [14] showed how to reduce the problem of maintaining Euler tours to that of supporting splits and merges of linked lists, and finding a minimum weight replacement (MWR) edge in the case of deleting an edge in $G$ that is also in $F$. While supporting operations on lists is generally straightforward, being able to find a MWR edge is the challenging aspect. Nevertheless, lists turns out to be very convenient for parallelization since different processors can focus on different parts of the list.

Chunks and LSDS.. In order to be able to manipulate the lists efficiently, we partition each list into chunks of size $K=O(\sqrt{n \log n})$, so that there are $J=O(\sqrt{n / \log n})$ chunks. The lists contain copies of vertices as they appear in the Euler tour, and so, by our assumptions, every chunk $c$ has as most $O(K)$ edges touching vertices with copies in $c$. Throughout the execution of the algorithm chunks are merged and split, either due to a list splitting at a chunk or in order to guarantee that every chunk is of size at most $O(K)$. Above the chunks, we use a tree-like data structure, called the list sum data structure (LSDS) that has at most $J$ leaves (one for each chunk) and height $O(\log J)$.

The basic idea is to separately aggregate useful information for finding MWR edges from all of the elements in each chunk, and then use the LSDS to aggregate all of the information from all of the chunks. The information stored for a chunk $c$ is an array $C A d j_{c}$ of size $J$ that stores the connectivity information between $c$ and every other chunk. The array stores one entry for each chunk such that the $i$ 'th entry in all of these arrays represents the chunk with id $i$. This information stored in $C A d j_{c}$ is derived from the at most $O(K)$ edges touching vertices that have a copy in $c$. The information stored at an LSDS tree vertex $u$ is an array $C A d j_{u}$ of size $J$ which stores the aggregate of all of the chunk information of chunks in the subtree of $u$.

When a chunk $c$ is the outcome of either a split or merge of chunks, $C A d j_{c}$ is updated by scanning the elements of $c$ in $O(K)$ worst-case time (since each chunk contains at most $O(K)$ elements). Then, for every other chunk $c^{\prime}$ the connectivity information between $c^{\prime}$ and $c$ that needs to be stored in $C A d j_{c^{\prime}}$ is derived from $C A d j_{c}$. Since there are $O(J)$ chunks, this takes $O(J)$ worst-case time. The information in $C A d j_{c}$ is then propagated up the LSDS tree to the ancestors of $c$, spending $O(J)$ worst-case time per ancestor for a total of $O(J \log J)=O(K)$ worst-case time. Finally, the entries corresponding to $c$ in all of the vertices of the LSDS tree are updated by scanning the LSDS tree while only accessing the entries in the arrays that correspond to $c$. This last part spends $O(1)$ worst-case time per tree vertex for a total of $O(J)$ worst-case time. Thus, the cost of merging and splitting chunks ends up being $O(J+K)=O(\sqrt{n \log n})$.

In addition to the splitting and merging of chunks, the algorithm will sometimes need to split or merge LSDS structures. These splits and merges are standard tree operations, and each such tree operation touches $O(\log n)$ tree-vertices. The time cost is dominated by updating the $C A d j$ arrays (of size $O(J)$ each) of the vertices touched during the tree operations, for a total of $O(\sqrt{n \log n})$ worst-case time.

Finding a MWR edge. The method for finding a MWR edge is to find the lightest edge connecting the vertices with copies in one list $L_{1}$ and the vertices with copies in a second list $L_{2}$. We remark that there are some crucial details of this process which we skip in the overview description here.

The algorithm uses the $C A d j$ array stored at the root of the LSDS for $L_{2}$, which specifically contains the connectivity information between the chunks in $L_{2}$ and all other chunks. The MWR edge is found by scanning the $O(J)$ chunks of $L_{1}$, and for each such chunk $c$ we look at the entry corresponding to $c$ in the $C A d j$ array of the root of $L_{2}$. The chunk in $L_{1}$ that has the smallest entry in the $C A d j$ array contains the lightest edge connecting $L_{1}$ and $L_{2}$, and the algorithm scans all of the $O(K)$ edges touching vertices with copies in that chunk in order to find the MWR edge. The entire process costs $O(J+K)=O(\sqrt{n \log n})$ worst-case time.

Parallel dynamic MSF.. One of the advantages of our new sequential MSF algorithm is that it leads to an improved parallel dynamic MSF algorithm. The reason for the time cost being $O(\sqrt{n \log n})$ in the sequential algorithm is due to scanning all of the elements in a chunk, scanning all of the chunks of a list, and scanning all of the vertices in an LSDS. By utilizing several tournament like trees, we show how all of these tasks can be executed in parallel, with a cost of $O(\log n)$ parallel worst-case time.

Parallel sparsification. The sparsification method of Eppstein et al. [4] allows one to reduce the dependency of the sequential time cost for dynamic MSF in terms of the number of edges. In particular, this method admits a conversion of any algorithm for solving dynamic MSF with polynomial sequential time cost $f(m)$ to be reduced to a time cost of $f(n)$ by focusing on the special case in which $m=O(n)$. Roughly speaking, the method uses a tree based data structure that has $O(\log n)$ levels where the number of edges associated with a tree vertex at level $i$ is $n / 2^{i}$. Each update necessitates at most one update at each level and so the total time cost becomes $\sum_{i=1}^{O(\log n)} f\left(n / 2^{i}\right)$. 
Unfortunately, the sequential sparsification does not transfer immediately to the parallel setting. In the sequential algorithm of Section 2 the time cost on a graph with $O\left(n^{\prime}\right)$ edges is $f\left(n^{\prime}\right)=$ $O\left(\sqrt{n^{\prime} \log n^{\prime}}\right)$, and so using the sparsification method we have that $\left.\sum_{i=1}^{O(\log n)} \sqrt{\left(n / 2^{i}\right) \log \left(n / 2^{i}\right)}\right)=O(\sqrt{n \log n})$. However, in the parallel algorithm, since $f\left(n^{\prime}\right)=O\left(\log n^{\prime}\right)$ we have that the total cost is $\sum_{i=1}^{O(\log n)} \log \left(n / 2^{i}\right)=O\left(\log ^{2} n\right)$. This adds an $O(\log n)$ factor to the cost.

Ferragina [5] introduced a parallel technique to apply the sparsification data structure of Eppstein et al. [3] to any parallel algorithm solving the fully dynamic MSF problem, with a $O\left(\log \frac{m}{n}\right)$ factor to the total work. However, we are interested in a parallel sparsification method that does not increase any of the asymptotic costs of the dynamic MSF data structure. Thus, we introduce an augmentation of the Eppstein et al. [4] sparsification method which achieves this goal. This augmentation requires a more detailed description of how the spasification data structure works, which we detail in the full version [15].

\subsection{Other Related Work}

Related work on sequential dynamic MSF.. In the sequential setting, the fastest deterministic worst-case algorithm for dynamic MSF has an $O(\sqrt{n})$ worst-case update time [4, 6]. Henzinger and King [8] showed a deterministic algorithm for dynamic MSF with amortized update time of $O\left(n^{1 / 3} \log n\right)$. Holm et al. [9] designed another deterministic algorithm which has an amortized update time of $O\left(\log ^{4} n\right)$, and later Holm et al [10] improved the amortized update time to $O\left(\frac{\log ^{4} n}{\log \log n}\right)$. When allowing randomization, Wulff-Nilsen [25] gave a randomized Las-Vegas algorithm for dynamic MSF with an expected worst-case update time of $O\left(n^{\frac{1}{2}-\epsilon}\right)$ for some constant $\epsilon>0$, and Nanongkai et al. [18] gave a randomized Las-Vegas algorithm with an expected worst-case update time of $n^{O(\log \log \log n / \log \log n)}$. Pătraşcu et al. [19] proved a lower bound of $\Omega(\log n)$ update time for dynamic connectivity, which is also a lower bound for dynamic MSF.

Related work on sequential dynamic connectivity. Notice that an algorithm for dynamic MSF is also an algorithm for dynamic connectivity. However, often there are faster dynamic connectivity algorithms. For the related dynamic connectivity problem, as mentioned above, the fastest deterministic worst-case time algorithm has an $O\left(\sqrt{\frac{n(\log \log n)^{2}}{\log n}}\right)$ worst-case update time [14]. When allowing randomization, Kapron, King, and Mountjoy [13] gave a Monte Carlo randomized structure with update time $O\left(c \log ^{5} n\right)$ and one-sided error probability $n^{-c}$. The update time was later improved to $O\left(c \log ^{4} n\right)$ independently by Gibb et al. [7] and Wang [23]. When allowing amortization Wulff-Nilsen [24] discovered a deterministic data structure with $O\left(\log ^{2} n / \log \log n\right)$ amortized update time. Recently, Huang et al. [11] showed that if both randomization and amortization are allowed then dynamic connectivity can be solved in expected $O\left(\log n(\log \log n)^{2}\right)$ amortized time. Recently, Nanongkai and Saranurak [17] presented a randomized Las-Vegas algorithm for dynamic MSF with an expected worst-case update time of $O\left(n^{\frac{1}{2}-\epsilon}\right)$ for some constant $\epsilon>0$.

\subsection{Organization}

In Section 2 we give a formal detailed description of the sequential dynamic MSF algorithm proving Theorem 1.2. In Section 3 we give a formal detailed description of the EREW PRAM dynamic MSF algorithm for sparse graphs, thereby proving Theorem 1.1 for the special case of $m=O(n)$. Due to space considerations, the description of how to extend the sparsification technique of Eppstein et al. [4] to the EREW PRAM model, without affecting the costs, is given in the full version [15]. This technique completes the proof of Theorem 1.1 for general graphs.

\section{SEQUENTIAL DYNAMIC MSF - PROOF OF THEOREM 1.2}

The goal of this section is to present a deterministic sequential algorithm for dynamic MSF in sparse graphs, where $m=O(n)$. Theorem 1.2 is proved by applying the sparsification technique of Eppstein et al. [4] to the presented algorithm, allowing the algorithm to also work for general graphs, without changing the time cost.

\subsection{Preliminaries}

Each tree $T$ in the MSF is represented as an Euler tour Euler $(T)$, and each Euler tour (which is a list of edges) is stored as a list of vertices whose order is defined by the Euler tour such that every two adjacent vertices in the list represent an edge in the tour. We say that an edge in the MSF is a tree edge. When a new edge $e=(u, v)$ is added to the graph, if $e$ connects two different trees in the MSF then $e$ becomes a tree edge, and the two corresponding Euler tours are merged. Otherwise, let $e^{\prime}$ be the heaviest edge on the path between $u$ and $v$ in the MSF prior to the update. If $w(e)<w\left(e^{\prime}\right)$ then the algorithm removes $e^{\prime}$ from the MSF and adds $e$ instead, thereby rearranging Euler tours. When a non-tree edge is deleted, there are no changes to any of the Euler tours. However, when a tree edge is deleted, the algorithm looks for a Minimum Weight Replacement (MWR) edge to reconnect the MSF, thereby rearranging Euler tours. Thus, the task of rearranging Euler tours is reduced to surgical operations of splitting or merging lists, as expressed in the following lemma (proven in [14]).

Lemma 2.1 ([14], Lemma 2.1). Let $G$ be an undirected graph with $M S F F$. Let $T \in F$ be a MST in $F$ that contains a tree edge e. If e is deleted, and $T=T_{0} \cup e \cup T_{1}$, then $\operatorname{Euler}\left(T_{0}\right)$ and $\operatorname{Euler}\left(T_{1}\right)$ are constructed from Euler $(T)$ with $O(1)$ surgical operations. In the opposite direction, from Euler $\left(T_{0}\right)$ and $\operatorname{Euler}\left(T_{1}\right)$, Euler $\left(T_{0} \cup e \cup T_{1}\right)$ is constructed with $O(1)$ surgical operations. It takes $O(1)$ worst-case time to determine which surgical operations to perform.

Notice that during the sequence of surgical operations (of splitting or merging lists) that take place during splitting or merging Euler tours (as expressed in Lemma 2.1), the lists of vertices may temporarily not be valid Euler tours.

The discussion above together with Lemma 2.1 reduces the dynamic MSF problem to the following three subproblems: (1) finding the heaviest edge between two given vertices in a dynamic forest, (2) finding a MWR edge, and (3) implementing surgical operations on lists. The first subproblem is solved by applying the dynamic tree data structure of Sleator and Tarjan [20], which costs $O(\log n)$ 
worst-case time per forest update or path query. The rest of the description focuses on solving the last two subproblems. We emphasize that implementing the surgical operations on lists can be done in a straightforward manner costing $O(1)$ worst-case time per operation, but such an implementation does not seem to support an efficient implementation of finding a MWR edge.

\subsection{The Data Structure}

Principal copies. For a graph vertex $u$ there could be several copies of $u$ in the list of vertices containing $u$ (since the purpose of the list is to represent an Euler tour). The algorithm designates one copy to be the principal copy of $u$, denoted by $p c_{u}$, and vertex $u$ stores a bidirectional pointer to $p c_{u}$.

Chunks. Each list of vertices is partitioned into consecutive chunks of vertices, so that each chunk $c$ contains $O(K)$ vertices and there are $O(K)$ edges incident to vertices whose principal copy is in $c$, for a carefully chosen parameter $K$. We say that an edge is adjacent to or touches chunk $c$ if the edge touches a graph vertex whose principal copy is in $c$. The algorithm maintains Invariant 1 for all chunks (recall that the maximum degree in the graph is 3):

INVARIANT 1. For chunk $c$ let $n_{c}$ be the sum of the number of vertices in $c$ and the number of edges adjacent to $c$. Then $n_{c} \leq 3 K$, and if $c$ is not the only chunk in the list containing $c$ then $K \leq n_{c}$.

Notice that when Invariant 1 is violated, a standard technique of merging and/or splitting $O(1)$ adjacent chunks is used in order to restore the invariant. We describe the process for merging and splitting chunks in the proof of Lemma 2.2 .

We assume for now that every list contains at least two chunks. The special case of lists containing only one chunk is addressed in the full version [15].Thus, we denote the maximum number of possible chunks by $J=O(n / K)$. Each chunk $c$ is assigned a unique id $i d_{c} \in[J]$, and each vertex in $c$ stores $i d_{c}$. In order to support quick lookups of chunks, the algorithm stores a $J$ sized array called chunks such that chunks $\left[i d_{c}\right]=c$. Each chunk $c$ maintains two $J$-length vectors called $C A d j_{c}$ and $M e m b_{c} . C A d j_{c}\left[i d_{c^{\prime}}\right]$ contains the minimum weight of an edge $(u, v)$ such that $p c_{u}$ is in $c$ and $p c_{v}$ is in $c^{\prime}$. If no such edge exists then we denote $C A d j_{c}\left[i d_{c^{\prime}}\right]=\infty$. In the $M e m b_{c}$ vector, all of the entries are set to 0 except for the entry at $i d_{c}$ which is set to 1 .

The LSDS.. For each list $L$, the data structure stores a list sum data structure (LSDS) which is implemented as a 2-3 tree whose leaves correspond, in order, to the chunks of $L$. The LSDS supports logarithmic worst-case time inserts, deletes, splits and joins. Each internal vertex $z$ maintains two $J$-length vectors, $M e m b_{z}$ and $C A d j_{z}$. $M e m b_{z}$ is the entry-wise OR of all the $M e m b$ vectors of chunks contained in leaves in the subtree of $z . C A d j_{z}$ is the entry-wise minimum of all the $C A d j$ vectors of chunks contained in leaves in the subtree of $z$, as shown in Figure 1.

In order to efficiently perform surgical operations on lists, we describe an efficient implementation for splitting and merging chunks (Section 2.3) and an efficient implementation of LSDS operations (Section 2.4). We then use these implementations to show how to efficiently implement the surgical operations and how to find a MWR edge (Section 2.5).

\subsection{Splitting and Merging Chunks}

Lemma 2.2. There exists a data structure on chunks that supports splits and merges such that each operation costs $O(J+K)$ worst-case time.

Proof. Splitting. Splitting a chunk $c$ can happen for one of two reasons: (1) either the list containing $c$ needs to be split at a given vertex $u$ that is in $c$, or (2) $n_{c}>3 K$ thereby violating Invariant 1 . In the second case, the split location is located by scanning $c$ in $O(K)$ worst-case time. Thus, we assume from now that the algorithm knows the split location $u$.

Splitting the list of vertices in $c$ at vertex $u$ takes $O(1)$ worst-case time. Let $c_{1}$ and $c_{2}$ be the resulting chunks where $c_{1}$ contains the first part of the list of vertices from $c$ and $c_{2}$ contains the second part. The algorithm sets $i d_{c_{1}}=i d_{c}$ and allocates a new (unique) id for $c_{2}$. Next, the algorithm scans all of the vertices in $c_{2}$ and updates their chunk id. The new $C A d j$ arrays for $c_{1}$ and $c_{2}$ are created by iterating over all edges adjacent to $c_{1}$ and $c_{2}$, respectively, in $O(K)$ worst-case time. Finally, for each chunk $c^{\prime}$, the algorithm updates $C A d j_{c^{\prime}}\left[i d_{c_{1}}\right]$ to be $C A d j_{c_{1}}\left[i d_{c^{\prime}}\right]$ and $C A d j_{c^{\prime}}\left[i d_{c_{2}}\right]$ to be $C A d j_{c_{2}}\left[i d_{c^{\prime}}\right]$, which takes $O(J)$ worst-case time. Thus, the cost for splitting a chunk is $O(J+K)$ worst-case time.

Merging. Merging the lists of vertices of adjacent chunks $c_{1}$ and $c_{2}$ takes $O(1)$ worst-case time. Let $c$ denote the resulting chunk containing the concatenation of the two lists. The algorithm sets $i d_{c}=i d_{c_{1}}$, and in $O(K)$ worst-case time the algorithm scans all of the vertices in $c$ in order to update their chunk id. The new $C A d j$ array for $c$ is created by iterating over all edges incident to $c$ in $O(K)$ worst-case time. Finally, for each chunk $c^{\prime}$, the algorithm updates $C A d j_{c^{\prime}}\left[i d_{c}\right]$ to be $C A d j_{c}\left[i d_{c^{\prime}}\right]$ and sets $C A d j_{c^{\prime}}\left[i d_{c_{2}}\right]=\infty$, which takes $O(J)$ worst-case time. Thus, the cost for merging two adjacent chunks $O(J+K)$ worst-case time.

\subsection{Implementing The LSDS Operations}

The LSDS supports the following operations:

- LSInsert $\left(c, c^{\prime}\right)$ - Add a new leaf for chunk $c$ after chunk $c^{\prime}$.

- LSDelete $(c)$ - Destroy chunk $c$.

- LSJoin $\left(L S_{1}, L S_{2}\right)$ - Concatenate two lists of chunks represented by LSDS $L S_{1}$ and LSDS $L S_{2}$.

- LSSplit(c) - Split the LSDS at chunk $c$.

- UpdateAdj $(c)$ - (Takes place immediately after an update to $C A d j_{c}$ and $C A d j_{c^{\prime}}\left[i d_{c}\right]$ for all chunks $c^{\prime} \neq c$.) Update the $C A d j$ vectors for ancestors of $c$ in the LSDS, and update the $i d_{c}$ 'th entry of the $C A d j$ vectors for all of the vertices in the LSDS.

LEMMA 2.3. There exists an implementation of the LSDS where each of the operations LSInsert, LSDelete, LSJoin, LSSplit and UpdateAdj take $O(J \log J)$ worst-case time.

Proof. All operations except for UpdateAdj. Basic tree operations on the LSDS, including access, insertion, deletion, splitting and joining cost $O(\log J)$ worst-case time each (since each LSDS supports at most $J$ chunks). Thus, each basic tree operation touches at most $O(\log J)$ vertices in the tree. For every vertex $z$ in the LSDS, updating a single entry in $C A d j_{z}$ or $M e m b_{z}$ costs $O(1)$ worst-case time (since the number of children is $O(1)$ ), and so updating the 

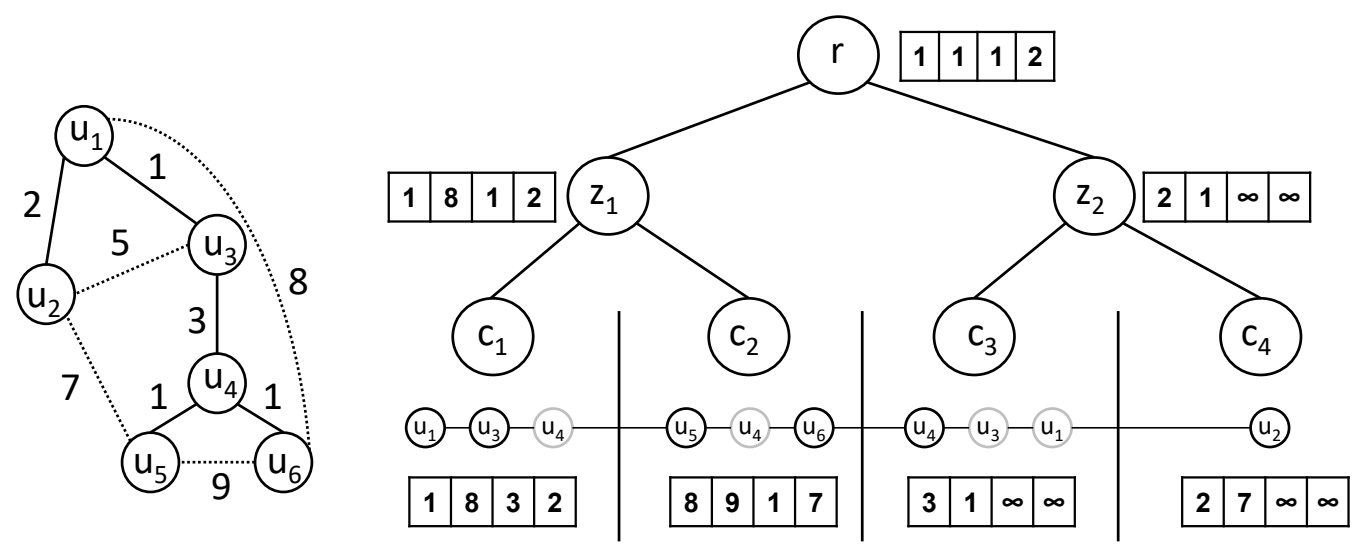

Figure 1: The left part of the figure is a graph where the tree edges are solid edges and the non-tree edges are the dotted edges. The right part of the figure is a LSDS tree whose leaves are chunks of the list of vertices representing the Euler tour for the graph. Each chunk contains a sublist of the Euler tour. The principal copies are the black vertices. The arrays in each chunk and next to internal tree vertices are the $C A d j$ vectors.

$C A d j$ and $M e m b$ arrays for the $O(\log J)$ vertices touched during a basic tree operation takes at most $O(J \log J)$ worst-case time.

Operation UpdateAdj. Updating the CAdj vectors in the path from the leaf representing $c$ to the root takes $O(J \log J)$ worst-case time. Updating the $i d_{c}$ 'th entry of every $C A d j$ array in the tree takes $O(J)$ worst-case time (since the 2-3 tree contains at most $O(J)$ vertices). Thus, the total cost of UpdateAdj is $O(J \log J)$ worst-case time.

\subsection{Surgical Operations}

LEMMA 2.4. There exists an algorithm in which each surgical operation on lists costs $O(J \log J+K)$ worst-case time and finding a MWR edge costs $O(J+K)$ worst-case time.

Proof. Splitting a list. Suppose we split list $L$ at vertex $u$ of chunk $c$ into two parts, $L_{1}$ and $L_{2}$. Let $L S$ be the LSDS representing $L$. The algorithm splits $c$ at vertex $u$ into $c_{1}$ and $c_{2}$, inserts $c_{2}$ into $L S$ after the leaf representing $c_{1}$ and calls $\operatorname{UpdateAdj}\left(c_{1}\right)$ and $\operatorname{UpdateAdj}\left(c_{2}\right)$ in order to update all $C A d j$ vectors in $L S$. Next, the algorithm splits $L S$ into $L S_{1}$ and $L S_{2}$, where the last chunk of $L S_{1}$ is $c_{1}$ and the first chunk of $L S_{2}$ is $c_{2}$. If Invariant 1 is violated at either $c_{1}$ or $c_{2}$, then the algorithm executes $O(1)$ splits and merges (followed by $O(1)$ LSDS operations) on $c_{1}$ or $c_{2}$ together with their adjacent chunks in $L_{1}$ or $L_{2}$, respectively, thereby preserving Invariant 1 . Thus, splitting a list costs $O(J \log J+K)$ worst-case time.

foining two lists. Suppose we join two lists, $L_{1}$ and $L_{2}$, into a single list $L$. Let $L S_{1}\left(L S_{2}\right)$ be the corresponding LSDS of $L_{1}\left(L_{2}\right)$. The algorithm calls $L S J o i n\left(L S_{1}, L S_{2}\right)$ to merge $L S_{1}$ and $L S_{2}$ into $L S$, which costs $O(J \log J)$ worst-case time.

Finding a MWR edge. Notice that the algorithm looks for a MWR edge between two Euler tours $E T_{1}$ and $E T_{2}$ only immediately after splitting Euler tour $E T$ into $E T_{1}$ and $E T_{2}$. Let $L S_{1}\left(L S_{2}\right)$ be the LSDS corresponding to the list of $E T_{1}\left(E T_{2}\right)$. Let $r_{1}$ and $r_{2}$ be the roots of $L S_{1}$ and $L S_{2}$, respectively.
The algorithm constructs an array $\gamma$ of length $J$ in $O(J)$ worstcase time such that if $M e m b_{r_{2}}[i]=0$ then $\gamma[i]=\infty$, and otherwise $\gamma[i]=C A d j_{r_{1}}[i]$. Thus, $\gamma\left[i d_{c}\right]<\infty$ if and only if there exists some edges between vertices in $E T_{1}$ and vertices in chunk $c$ (which must be in $E T_{2}$ ). Moreover, if $\gamma\left[i d_{c}\right]<\infty$ then the weight of the minimum weight edge between $E T_{1}$ and chunk $c$ is $\gamma\left[i d_{c}\right]$. Let $\hat{c}=\arg \min _{\text {chunk } c}\left\{\gamma\left[i d_{c}\right]\right\}$. Thus, the minimum weight edge between $E T_{1}$ and $E T_{2}$ touches a graph vertex $u$ such that $p c_{u} \in \hat{c}$. The algorithm computes $i d_{\hat{c}}$ in $O(J)$ worst-case time by scanning $\gamma$ and looking for the smallest entry. Then, the algorithm scans all of the $O(K)$ edges touching $\hat{c}=\operatorname{chunks}\left[i d_{\hat{c}}\right]$, and for each such edge $e=(u, v)$ where $p c_{u} \in \hat{c}$, the algorithm verifies whether the chunk $c_{v}$ containing $p c_{v}$ is in $L S_{1}$ or not by looking at $M e m b_{r_{1}}\left[i d_{c_{v}}\right]$. Finally, the algorithm picks the lightest edge that passes the verification. Thus, the total cost of finding the MWR edge is $O(J+K)$ worst-case time.

\subsection{Graph Updates}

Proof of Theorem 1.2. Edge insertion. Suppose we insert a new edge $e=(u, v)$ with weight $w(e)$ to the graph. Let $c_{1}$ and $c_{2}$ be the chunks containing $p c_{u}$ and $p c_{v}$, respectively, and let $L S_{1}$ and $L S_{2}$ be the LSDSes containing $c_{1}$ and $c_{2}$, respectively. The algorithm begins by updating $C A d j_{c_{1}}\left[i d_{c_{2}}\right]$ and $C A d j_{c_{2}}\left[i d_{c_{1}}\right]$. Next, the algorithm calls UpdateAdj $\left(c_{1}\right)$ and UpdateAdj $\left(c_{2}\right)$ in order to update the $C A d j$ vectors in $L S_{1}$ and $L S_{2}$. In case of a violation to Invariant 1 , the algorithm executes $O(1)$ splits and merges on $c_{1}$ or $c_{2}$ together with their respective adjacent chunks, followed by $O(1)$ LSDS operations.

If $L S_{1} \neq L S_{2}$ then $u$ and $v$ are in different Euler tours, and so by Lemma 2.1 a series of $O(1)$ surgical operations takes place in order to merge the two Euler tours containing $u$ and $v$ into a single Euler tour. The algorithm also adds $e$ to the dynamic tree structure of Sleator and Trajan [20] in $O(\log n)$ worst-case time.

If $L S_{1}=L S_{2}$, then $u$ and $v$ are in the same Euler tour. In this case, the algorithm uses the dynamic tree structure to locate the heaviest edge $e^{\prime}$ on the path from $u$ to $v$ in the current MSF. Finding $e^{\prime}$ takes $O(\log n)$ worst-case time. If $w(e)<w\left(e^{\prime}\right)$ then the algorithm 
removes $e^{\prime}$ from the MSF, inserts $e$ into the MSF, and updates the dynamic tree structure which $\operatorname{costs} O(\log n)$ worst-case time. Thus, the total worst-case time for inserting an edge is $O(J \log J+K+$ $\log n)$.

Edge deletion. Suppose we delete edge $e=(u, v)$ from the graph. Let $c_{1}$ be the chunk containing $p c_{u}$ and let $c_{2}$ be the chunk containing $p c_{v}$. Let $L S$ be the LSDS containing $c_{1}$ and $c_{2}$. The algorithm begins by updating $C A \operatorname{Adj}_{c_{1}}\left[i d_{c_{2}}\right]$ and $C A d j_{c_{2}}\left[i d_{c_{1}}\right]$ in $O(K)$ worstcase time by scanning all edges touching $c_{1}$. Next, the algorithm calls UpdateAdj $\left(c_{1}\right)$ on $L S$ to update all $C A d j$ vectors in $L S$. If $e$ is a tree edge, then the algorithm first removes $e$ from the dynamic tree structure in $O(\log n)$ worst-case time, and then executes a series of $O(1)$ surgical operations in order to split the Euler tour containing $u$ and $v$. Let $E T_{u}$ and $E T_{v}$ be the resulting two Euler tours containing $u$ and $v$, respectively. Finally, the algorithm looks for a MWR edge between $E T_{u}$ and $E T_{v}$ in $O(J+K)$ worst-case time, and if such an edge $e^{\prime}$ is found, then the algorithm adds $e^{\prime}$ to the dynamic tree structure and executes another series of $O(1)$ surgical operations reconnecting $E T_{u}$ and $E T_{v}$. Thus, the cost of deleting an edge is $O(J \log J+K+\log n)$ worst-case time.

Time cost. By setting $K=O(\sqrt{n \log n})$ and since $J=O(n / K)$, the insertion and deletion costs become $O(J \log J+K+\log n)=$ $O(\sqrt{n \log n})$ worst-case time.

\section{PARALLEL DYNAMIC MSF ON SPARSE GRAPHS}

In this section we prove the following theorem.

THEOREM 3.1. There exists a deterministic algorithm for the $d y$ namic MSF problem in the EREW PRAM model on sparse graphs with $m=O(n)$ edges that uses $O(\sqrt{n})$ processors and has a parallel worstcase update time of $O(\log n)$. The resulted work of the algorithm is $O(\sqrt{n} \log n)$.

The new parallel sparsification technique (details in the full version [15]), extends Theorem 3.1 to work for general graphs, thereby proving Theorem 1.1.

The data structure. The algorithm uses the same data structure as described in Section 2.2 with three changes. The first change is that for each chunk $c$, the list of vertices in $c$ is augmented with a balanced 2-3 tree, denoted by $B T_{c}$, whose leaves are elements of the list that are in $c$. The height of $B T_{c}$ is $O(\log K)$. Each vertex $v$ in $B T_{c}$ stores an edge counter $e c_{v}$ which is the total number of edges incident to graph vertices whose principal copy is in the subtree of $v$; see Figure 2. The order of leaves in $B T_{c}$ together with an order of the at most 3 edges incident to each graph vertex whose principal copy is in $c$ defines an order on the edges touching $c$.

The second change is due to the requirements from the EREW PRAM model. In particular, for any chunk $c$, we cannot support constant worst-case time access to the entries of $C A d j_{c}$ (or Memb $b_{c}$ ) in parallel through a single pointer from $c$ to the array $C A d j_{c}$, due to the exclusive reading requirement. Instead, we use a two dimensional matrix $C$ of size $J \times J=O(n)$ (at the end of this section we set $K=\sqrt{n}$ ) such that the entries of the $j$ 'th row of $C$ are exactly the entries of $C A d j_{c}$ where $i d_{c}=j$. From now on, we let $C A d j_{c}[i]$ denote $C\left[i d_{c}, i\right]$. We also use the same exact method for Memb arrays.
The third change, which is also due to the exclusive reading requirement, is in the $C A d j$ and Memb arrays in the LSDS. Instead of using one tree LSDS $L S$, we now use $J$ trees $S_{1}, S_{2}, \ldots, S_{J}$ for each LSDS, where the $j$ 'th tree corresponds to the chunk with id $j$. For chunk $c$, the $i d_{c}$ 'th leaf of $S_{j}$ contains both $C A d_{c}[j]$ and $M e m b_{c}[j]$. We also store a pointer from $C A d j_{c}[j]$ and $M e m b_{c}[j]$ to the $i d_{c}$ 'th leaf of $S_{j}$, thereby providing direct access to that leaf. Finally, in order to provide direct access to the root of each $S_{j}$, we store a matrix of size $J \times J=O(n)$ where the $(j, i)$ entry contains a pointer to the root of $S_{i}$ used in the $j$ 'th LSDS.

As in the sequential algorithm, the special case of lists containing only one chunk in the new parallel algorithm is addressed in the full version [15].

Assigning edges. Our algorithm will often perform the task of assigning a different processor to each edge touching chunk $c$. This assignment is implemented by a parallel operation $\operatorname{get} \mathrm{dge} e_{c}(k)$ in which processor $p_{k}$ accesses the $k$ 'th edge incident to chunk $c$. The operation getEdge $(k)$ uses the edge counters in $B T_{c}$ together with an array vertex of size $3 K$ where each entry is a pointer to a vertex in $B T_{c}$. We describe the implementation from the perspective of processor $p_{k}$ for $1 \leq k \leq 3 K$. We emphasize that in order to implement $g e t E d g e_{c}(k)$, only $p_{1}$ will require access to $c$

Let $\operatorname{root}_{c}$ be the root of $B T_{c}$. The implementation has $h$ phases where $h=O(\log K)$ is the height of $B T_{c}$. Processor $p_{k}$ participates in the $i$ 'th phase if and only if vertex $[k] \neq N U L L$ at the beginning of the $i$ 'th phase. Moreover, the participating processors in each phase are assigned to different vertices in $B T_{c}$. In particular, processor $p_{k}$ is assigned to the vertex $v=$ vertex $[k]$ with the guarantee that the rank of the rightmost edge in the subtree of $v$ is $k$.

To initialize the process, each $p_{k}$ sets vertex $[k]=N U L L$ and if $k=1$ then $p_{k}$ sets vertex $\left[e c_{\text {root }_{c}}\right]=\operatorname{root}_{c}$. Now we begin the phases for $i=1,2, \ldots, h$. For the $i$ 'th phase, if vertex $[k]=v$, then $p_{k}$ accesses the at most 3 children of $v$ and looks at their edge counters. Based on these edge counters, $p_{k}$ computes in constant worst-case time the rank of the rightmost edge in each one of the subtrees of the children of $v$. If the rightmost edge in the subtree of a child $u$ of $v$ is $r$, then $p_{k}$ sets vertex $[r]=u$. Notice that $p_{k}$ necessarily sets vertex $[k]$ to be the rightmost child of $v$. After $h$ phases all of the vertices in vertex are leaves of $B T_{c}$, but some of the entries of vertex may still be set to NULL. An entry vertex $[k]=N U L L$ can occur due to one of two reasons: either $k$ is larger than the number of edges touching $c$, or the principal copy of the edge that $p_{k}$ is accessing is also the principal copy of another edge which is being accessed by a different processor. However, due to the invariant that the rank of the rightmost edge in the subtree of vertex $[k]$ is $k$ and the fact that the maximum degree in the graph is 3 , the principal copy that $p_{k}$ is looking for is either in vertex $[k+1]$ or vertex $[k+2]$. Thus, within 3 more steps, $p_{k}$ is able to access the principal copy and complete the task. Thus, the operation $g e t E d g e_{c}(k)$ costs $O(\log K)$ worst-case time.

\subsection{Splitting and Merging Chunks}

LEMMA 3.2. There exists an algorithm in the EREW PRAM model that supports splits and merges of chunks such that each operation costs $O(\log K)$ parallel worst-case time, using $O(J+K)$ processors. 


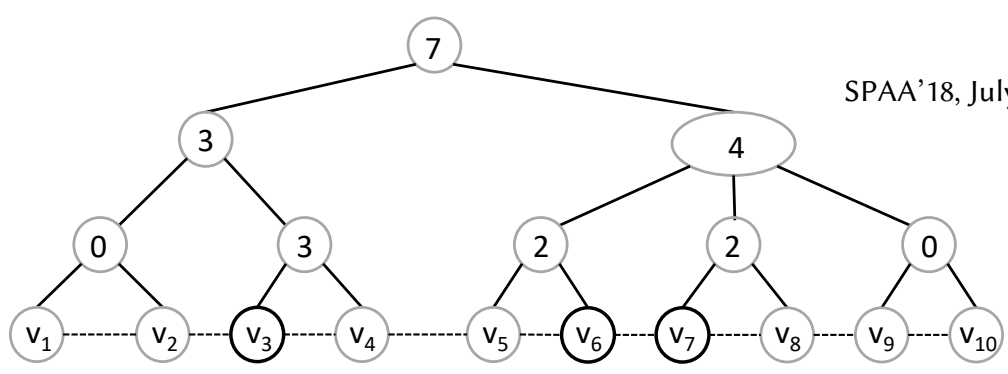

Figure 2: The 2-3 tree $B T_{c}$ built on the list of vertices inside chunk $c . v_{3}, v_{6}$ and $v_{7}$ are the principal copies inside chunk $c$, and the number of edges touching $v_{3}, v_{6}$ and $v_{7}$ is 3,2 , and 3 , respectively. The numbers inside the inner tree vertices are the edge counters.

Proof. Splitting. Recall that splitting a chunk $c$ can happen for one of two reasons: (1) either the list containing $c$ needs to be split at a given vertex $u$ that is in $c$, or (2) $n_{c}>3 K$ thereby violating Invariant 1 . In the second case, processor $p_{1}$ locates the split location in $O(\log K)$ worst-case time by traversing down $B T_{c}$ using the edge counters. Thus, we assume from now that the algorithm knows the split location $u$.

Processor $p_{1}$ splits $B T_{c}$ at vertex $u$ in $O(\log K)$ worst-case time. Let $c_{1}$ and $c_{2}$ be the resulting chunks where $c_{1}$ contains the first part of $B T_{c}$ and $c_{2}$ contains the second part. Processor $p_{1}$ sets $i d_{c_{1}}=i d_{c}$ and allocates a new (unique) id for $c_{2}$. We now focus on creating $C A d j_{c_{1}}$, since $C A d j_{c_{2}}$ is created in the same manner.

The sequential algorithm for constructing $C A d j_{c_{1}}$ (in the proof of Theorem 1.2) scans all of the $O(K)$ edges touching $c_{1}$. In the parallel setting, accessing all of the edges in parallel does not suffice since there could be several edges touching both $c_{1}$ and $\hat{c}$ for some other chunk $\hat{c}$, and the algorithm needs to store only the minimum weight of such an edge. To solve this issue we do the following.

The algorithm uses $J$ balanced binary tournament trees, denoted by $T_{1}, T_{2}, \ldots, T_{J}$, where each tree has $3 K$ leaves. Each vertex $z$ in $T_{j}$ stores a value $A_{z}$ initialized to $\infty^{1}$. The algorithm uses an iterative process for implementing a special tournament-like process. During the iterative process, each processor will initially be active until the processor decides to become inactive and no longer participates in the process. The iterative process implicitly uses an exclusiveassignment property which states that each participating processor is assigned to a vertex in some tree $T_{j}$ such that there are no two processors that are assigned to the same vertex. At the beginning of the $i$ 'th iteration the active processors are assigned to vertices whose height is $i-1$.

The initialization of the iterative process is as follows. For each $1 \leq k \leq 3 K$, processor $p_{k}$ sets itself as active and executes the getEdge $e_{c_{1}}(k)$ operation thereby gaining access to $e_{k}=\left(u_{k}, v_{k}\right)$, which is the $k$ 'th edge adjacent to chunk $c_{1}$. Assume without loss of generality that $p c_{u_{k}} \in c_{1}$. Let $c_{v_{k}}$ be the chunk such that $p c_{v_{k}} \in c_{v_{k}}$, and denote $i d=i d_{v_{v_{k}}}$. Processor $p_{k}$ assigns itself in $O(1)$ worstcase time (using a lookup table) to the $k$ 'th leaf of $T_{i d}$, denoted by $\ell_{k}$, and sets $A_{\ell_{k}}=w\left(e_{k}\right)$. Thus, the exclusive-assignment property holds.

Each iteration has four synchronous phases. Recall that only active processors continue to participate in the process.

- Phase 1. If $p_{k}$ is assigned to a vertex $z$ that is the left child of its parent parent $(z)$ then $p_{k}$ sets $A_{\text {parent }(z)}=w\left(e_{k}\right)$.

\footnotetext{
${ }^{1}$ Reusing and initializing a temporary data structure in the parallel setting is implemented by either using a timestamp for each word of memory or rolling back all of the memory changes after the operation completes, thereby allowing the cost analysis to ignore the initialization cost.
}

- Phase 2. If $p_{k}$ is assigned to a vertex $z$ that is the right child of its parent parent $(z)$ then: if $A_{\text {parent }}(z)>w\left(e_{k}\right)$ then $p_{k}$ sets $A_{\text {parent }(z)}=w\left(e_{k}\right)$ and otherwise $p_{k}$ becomes inactive.

- Phase 3. If $p_{k}$ participated in the first phase and $A_{\text {parent }(z)}<$ $w\left(e_{k}\right)$ then $p_{k}$ becomes inactive.

- Phase 4. If parent $(z)$ is the root of a tournament tree, then the iterative process ends. Otherwise, $p_{k}$ is assigned to parent $(z)$.

Notice that by the exclusive-assignment property we are guaranteed that during the first two phases no two processors are writing to the same location in memory at the same time. Also, we are guaranteed that if two processors are assigned to sibling vertices, then after the third phase the processor that is assigned to the lighter edge remains active, with ties favoring the left vertex, while the other processor becomes inactive. Thus, after the third phase, if $p_{k}$ is still active then there is no other active processor $p_{\hat{k}}$ that is currently assigned to $z$, and so after the fourth phase the exclusive-assignment property holds. At the end of the iterative process, the processor that is at the root of $T_{j}$ sets $C A d j_{c_{1}}[j]=A_{\operatorname{root}\left(T_{j}\right)}$.

Finally, for each chunk $c^{\prime}$, the algorithm sets $C A d j_{c^{\prime}}\left[i d_{c_{1}}\right]=$ $C A d j_{c_{1}}\left[i d_{c^{\prime}}\right]$ and sets $C A d j_{c^{\prime}}\left[i d_{c_{2}}\right]=C A d j_{c_{2}}\left[i d_{c^{\prime}}\right]$, which takes $O(1)$ parallel worst-case time using $O(J)$ processors. Thus, the cost for splitting a chunk is $O(\log K)$ parallel worst-case time, using $O(J+K)$ processors.

Merging. Processor $p_{1}$ merges $B T_{c_{1}}$ and $B T_{c_{2}}$ in $O(\log K)$ worstcase time. Let $c$ denote the resulting chunk containing the concatenation of the lists of vertices represented by $B T_{c_{1}}$ and $B T_{c_{2}}$. Processor $p_{1}$ sets $i d_{c}=i d_{c_{1}}$. The new $C A d j$ array for $c$ is created by performing an entry-wise minimum of $C A d j_{c_{1}}$ and $C A d j_{c_{2}}$ in $O(1)$ parallel worst-case time using $O(J)$ processors. Finally, for each chunk $c^{\prime}$, the algorithm sets $C A d j_{c^{\prime}}\left[i d_{c}\right]=C A d j_{c}\left[i d_{c^{\prime}}\right]$ and sets $C A d j_{c^{\prime}}\left[i d_{c_{2}}\right]=\infty$, which takes $O(1)$ parallel worst-case time using $O(J)$ processors. Thus, the cost for merging a chunk is $O(\log K)$ parallel worst-case time, using $O(J)$ processors.

\subsection{LSDS Operations}

LEMMA 3.3. There exists an implementation of the LSDS in the EREW PRAM model using $O(J)$ processors where each of the operations LSInsert, LSDelete, LSJoin, LSSplit and UpdateAdj takes $O(\log J)$ parallel worst-case time.

Proof. All operations except for UpdateAdj. Recall that in the proof of Lemma 2.3 each basic tree operation touches at most $O(\log J)$ vertices in the tree. We use a similar implementation as in Lemma 2.3, but now processor $p_{j}$ for $1 \leq j \leq J$ performs the basic tree operations on $S_{j}$. Thus, the total parallel worst-case time for each operation except for UpdateAdj is $O(\log J)$.

Operation UpdateAdj $(c)$. We again use a similar implementation as in Lemma 2.3, with the following changes. For each $S_{j}$, updating the path from the leaf representing $c$ to the root of $S_{j} \operatorname{costs} O(\log J)$ 
parallel worst-case time using $O(J)$ processors. In order to update $S_{i d_{c}}$, processor $p_{j}$ for $1 \leq j \leq J$ is responsible for handling the leaf representing chunks $[j]$, which is accessible through the pointer stored in $C A d_{j_{c h u n k s}[j]}\left[i d_{c}\right]$. We now need to sweep up $S_{i d_{c}}$ in parallel, starting from all of the leaves of $S_{i d_{c}}$. This process is described next.

The algorithm begins an iterative process where at the beginning of the $i$ 'th iteration there is a unique processer assigned to each vertex of height $i-1$ in $S_{i d_{c}}$. At the $i$ 'th iteration, suppose $p_{j}$ is assigned to vertex $z$ of height $i-1$. Then $p_{j}$ is reassigned to parent $(z)$ only if $z$ is the leftmost child of parent $(z)$. If $z$ is not the leftmost child of parent $(z)$, then $p_{j}$ halts. Thus, each vertex at height $i$ is assigned to exactly one processor. If $p_{j}$ did not halt then $p_{j}$ updates the value stored in parent $(z)$ in $O(1)$ worst-case time. The iterative process ends at the root, which happens after $O(\log J)$ steps. The parallel worst-case time cost per level is $O(1)$ and $O(\log J)$ worstcase time for the entire procedure. The number of processors used is $O(J)$.

\subsection{Surgical Operations}

LEMMA 3.4. There exists an algorithm in the EREW PRAM model in which each surgical operation on lists costs $O(\log J+\log K)$ parallel worst-case time using $O(J+K)$ processors and finding a MWR edge costs $O(\log J+\log K)$ parallel worst-case time using $O(J+K)$ processors.

Proof. The implementation of both splitting and merging lists remains the same as in the proof of Lemma 2.4, but this time applying Lemma 3.2 instead of Lemma 2.2. So the operation of splitting a list costs $O(\log K)$ parallel worst-case time using $O(J+K)$ processors, and the operation of merging two lists costs $O(\log J+\log K)$ parallel worst-case time using $O(J+K)$ processors.

Finding a MWR edge. The algorithm constructs the array $\gamma$, as defined in the proof of Lemma 2.4, but now processor $p_{j}$ for $j \in[J]$ computes $\gamma[j]$ in $O(1)$ parallel worst-case time by accessing the root of $S_{j}$ in each LSDS in constant worst-case time (using the lookup matrix). Let $\hat{c}=\arg \min _{\text {chunk } c}\left\{\gamma\left[i d_{c}\right]\right\}$. Recall that the minimum weight edge between $E T_{1}$ and $E T_{2}$ (as defined in the proof of Lemma 2.4) touches a vertex $u$ such that $p c_{u} \in \hat{c}$. The algorithm in the proof of Lemma 2.4 computes $i d_{\hat{c}}$ in $O(J)$ worstcase time by scanning $\gamma$ and finding the smallest entry. In the EREW PRAM model, the algorithm uses a tournament tree to find the smallest entry, which costs $O(\log J)$ parallel worst-case time using $O(J)$ processors. Next, processor $p_{k}$ for $k \in[3 K]$ accesses edge $e_{k}=g$ et $E d g e_{\hat{c}}(k)$. Let $e_{k}=(u, v)$ where $p c_{u} \in \hat{c}$. In the CREW PRAM model, processor $p_{k}$ verifies in $O(1)$ whether the chunk $c_{v}$ containing $p c_{v}$ is in $L S_{1}$ (as defined in the proof of Lemma 2.4) by looking at the Memb value in the root of $S_{i d_{c_{v}}}$ of $L S_{1}$. Using the reduction of [12], this process costs $O(\log K)$ worst-case time in the EREW model. Finally, the algorithm picks the lightest edge via a tournament tree algorithm whose participants are the processors whose edge passed the verification. The algorithm for finding the MWR edge takes $O(\log J+\log K)$ parallel worst-case time, using $O(J)$ processors.

\subsection{Graph Updates}

Proof of Theorem 1.1. Edge insertion. The algorithm for inserting an edge is the same as in the sequential algorithm in the proof of Theorem 1.2, but this time applying Lemmas 3.2, 3.3, and 3.4 instead of Lemmas 2.2, 2.3, and 2.4. The parallel worst-case update time is $O(\log J+\log K)$, by using $O(J+K)$ processors.

Edge deletion. The algorithm for deleting an edge is the same as in the sequential algorithm in the proof of Theorem 1.2, except for two changes: (1) the edge deletion algorithm applys Lemmas 3.2, 3.3, and 3.4 instead of Lemmas 2.2, 2.3, and 2.4, and (2) the new minimum weight edge connecting chunks $c_{1}$ and $c_{2}$ (as defined in the edge deletion operation in the proof of Theorem 1.2) is found in the EREW PRAM model by using a tournament tree which costs $O(\log K)$ parallel worst-case time using $O(K)$ processors. The parallel worstcase update time is $O(\log J+\log K)$, by using $O(J+K)$ processors.

Time cost. By setting $K=O(\sqrt{n})$, the insertion and deletion costs become $O(\log n)$ parallel worst-case time using $O(\sqrt{n})$ processors, for a total work of $O(\sqrt{n} \log n)$.

\section{CONCLUSION}

We described an algorithm for solving dynamic MSF on sparse graphs in the EREW PRAM model that uses $O(\sqrt{n})$ processors and has $O(\log n)$ worst-case update time. The resulted work of the algorithm is $O(\sqrt{n} \log n)$. By extending the sparsification technique to work in the EREW PRAM model (see [15]), the algorithm can be used for solving dynamic MSF on general graphs with the same complexities. Thus, the total work is $O(\sqrt{n} \log n)$. We leave open the task of designing a solution that has a parallel $O(\log n)$ worst-case update time, but only $O(\sqrt{n})$ work, thereby matching the amount of work used in the sequential solutions.

\section{ACKNOWLEDGMENTS}

This work is supported in part by ISF grant 1278/16. This project has received funding from the European Research Council (ERC) under the European Union Horizon 2020 research and innovation programme (grant agreement No 683064).

\section{REFERENCES}

[1] Ittai Abraham, David Durfee, Ioannis Koutis, Sebastian Krinninger, and Richard Peng. On fully dynamic graph sparsifiers. In IEEE 57th Annual Symposium on Foundations of Computer Science, (FOCS), pages 335-344, 2016.

[2] Sajal K. Das and Paolo Ferragina. An o(n) work EREW parallel algorithm for updating MST. In 2'nd Annual European Symposium on Algorithms, (ESA), pages 331-342, 1994.

[3] David Eppstein, Zvi Galil, Giuseppe F. Italiano, and Amnon Nissenzweig. Sparsification-a technique for speeding up dynamic graph algorithms (extended abstract). In 33rd Annual Symposium on Foundations of Computer Science, (FOCS), pages 60-69, 1992.

[4] David Eppstein, Zvi Galil, Giuseppe F. Italiano, and Amnon Nissenzweig. Sparsification - a technique for speeding up dynamic graph algorithms. F. ACM, 44(5):669-696, 1997.

[5] Paolo Ferragina. An EREW PRAM fully-dynamic algorithm for MST. In The 9th International Parallel Processing Symposium, (IPPS), pages 93-100, 1995.

[6] Greg N. Frederickson. Data structures for on-line updating of minimum spanning trees, with applications. SIAM 7. Comput., 14(4):781-798, 1985.

[7] D. Gibb, B. M. Kapron, V. King, and N. Thorn. Dynamic graph connectivity with improved worst case update time and sublinear space. CoRR, abs/1509.06464, 2015.

[8] Monika Rauch Henzinger and Valerie King. Maintaining minimum spanning trees in dynamic graphs. In Automata, Languages and Programming, 24th International Colloquium, (ICALP), pages 594-604, 1997. 
[9] Jacob Holm, Kristian de Lichtenberg, and Mikkel Thorup. Poly-logarithmic deterministic fully-dynamic algorithms for connectivity, minimum spanning tree, 2-edge, and biconnectivity. F. ACM, 48(4):723-760, 2001.

[10] Jacob Holm, Eva Rotenberg, and Christian Wulff-Nilsen. Faster fully-dynamic minimum spanning forest. In Algorithms - 23rd Annual European Symposium, (ESA), Proceedings, pages 742-753, 2015.

[11] Shang-En Huang, Dawei Huang, Tsvi Kopelowitz, and Seth Pettie. Fully dynamic connectivity in $O\left(\log n(\log \log n)^{2}\right)$ amortized expected time. In Proceedings of the Twenty-Eighth Annual ACM-SIAM Symposium on Discrete Algorithms, (SODA) pages 510-520, 2017.

[12] Joseph JtaJta. An introduction to parallel algorithms. In Addison-Wesley, 1992.

[13] Bruce M. Kapron, Valerie King, and Ben Mountjoy. Dynamic graph connectivity in polylogarithmic worst case time. In Proceedings of the Twenty-Fourth Annual ACM-SIAM Symposium on Discrete Algorithms, (SODA), pages 1131-1142, 2013.

[14] Casper Kejlberg-Rasmussen, Tsvi Kopelowitz, Seth Pettie, and Mikkel Thorup. Faster worst case deterministic dynamic connectivity. In 24th Annual European Symposium on Algorithms, (ESA), pages 53:1-53:15, 2016.

[15] Tsvi Kopelowitz, Ely Porat, and Yair Rosenmutter. Improved worst-case deterministic parallel dynamic minimum spanning forest. CoRR, abs/1805.06151, 2018.

[16] W. Liang and B.D. McKay. Fully dynamic maintenance of minimum spanning trees by using a sublinear number of processors. In Unpublished Manuscripts, 1994
[17] Danupon Nanongkai and Thatchaphol Saranurak. Dynamic spanning forest with worst-case update time: adaptive, las vegas, and $\mathrm{o}\left(\mathrm{n}^{1 / 2}-\epsilon\right)$-time. In Proceedings of the 49th Annual ACM SIGACT Symposium on Theory of Computing, (STOC), pages $1122-1129,2017$.

[18] Danupon Nanongkai, Thatchaphol Saranurak, and Christian Wulff-Nilsen. Dynamic minimum spanning forest with subpolynomial worst-case update time. In 58th IEEE Annual Symposium on Foundations of Computer Science, (FOCS), pages 950-961, 2017.

[19] Mihai Pătraşcu and Erik D. Demaine. Logarithmic lower bounds in the cell-probe model. SIAM 7. Comput., 35(4):932-963, 2006.

[20] Daniel Dominic Sleator and Robert Endre Tarjan. A data structure for dynamic trees. F. Comput. Syst. Sci., 26(3):362-391, 1983.

[21] Mikkel Thorup. Dynamic graph algorithms with applications. In 7th Scandinavian Workshop on Algorithm Theory, (SWAT), pages 1-9, 2000.

[22] Mikkel Thorup. Fully-dynamic min-cut. Combinatorica, 27(1):91-127, 2007.

[23] Zhengyu Wang. An improved randomized data structure for dynamic graph connectivity. CoRR, abs/1510.04590, 2015

[24] Christian Wulff-Nilsen. Faster deterministic fully-dynamic graph connectivity. In Proceedings of the Twenty-Fourth Annual ACM-SIAM Symposium on Discrete Algorithms, (SODA), pages 1757-1769, 2013.

[25] Christian Wulff-Nilsen. Fully-dynamic minimum spanning forest with improved worst-case update time. In Proceedings of the 49th Annual ACM SIGACT Symposium on Theory of Computing, (STOC), pages 1130-1143, 2017. 